University of Nebraska - Lincoln

DigitalCommons@University of Nebraska - Lincoln

USDA National Wildlife Research Center - Staff Publications
U.S. Department of Agriculture: Animal and Plant Health Inspection Service

2020

\title{
Influenza A Viruses in Peridomestic Mammals
}

J. Jeffrey Root

Susan A. Shriner

Follow this and additional works at: https://digitalcommons.unl.edu/icwdm_usdanwrc

Part of the Natural Resources and Conservation Commons, Natural Resources Management and Policy Commons, Other Environmental Sciences Commons, Other Veterinary Medicine Commons, Population Biology Commons, Terrestrial and Aquatic Ecology Commons, Veterinary Infectious Diseases Commons, Veterinary Microbiology and Immunobiology Commons, Veterinary Preventive Medicine, Epidemiology, and Public Health Commons, and the Zoology Commons

This Article is brought to you for free and open access by the U.S. Department of Agriculture: Animal and Plant Health Inspection Service at DigitalCommons@University of Nebraska - Lincoln. It has been accepted for inclusion in USDA National Wildlife Research Center - Staff Publications by an authorized administrator of DigitalCommons@University of Nebraska - Lincoln. 


\title{
Chapter 32
}

\section{Influenza A Viruses in Peridomestic Mammals}

\author{
J. Jeffrey Root and Susan A. Shriner
}

\begin{abstract}
During recent years, serological evidence has shown that a number of peridomestic mammals (e.g., those commonly found in or around human structures) are naturally exposed to influenza A viruses (IAVs). In addition, experimental studies have demonstrated that many of these species can successfully replicate several different IAVs, including IAVs of high consequence to public or agricultural health. The replication of some IAVs within this group of mammals could have implications for biosecurity associated with poultry production and live bird markets in some regions of the world. Given this evidence, the need for further study and understanding of the role that peridomestic mammals may play in IAV dynamics is increasingly being recognized. This chapter will provide a general overview on IAV associations in peridomestic mammals, especially as they pertain to avian IAVs, and provide some general views and guidelines for sampling these species in various situations.
\end{abstract}

Key words Mammal, Peridomestic, Synanthropic, Influenza A virus, Exposure, Transmission, Antibodies

\section{Introduction}

\subsection{Peridomestic Mammals}

A peridomestic mammal is a wild or feral mammal that can be found living within close proximity to humans or human structures or that utilizes resources associated with human altered landscapes. While we focus on wild, non-volant mammals in this chapter, feral cats, bats, and other species may be of interest. Peridomestic associations are often driven by sustenance, perceived sustenance, shelter, and/or predator avoidance (e.g., small rodents) in anthropogenically modified environments. Peridomestic mammals are distributed in a wide range of habitats from highly urbanized landscapes, rural farm settings, to lightly modified habitats associated with the temporary shelters of pastoralists. Further, a mammal can be considered peridomestic in some situations, but the same species, while living in its natural environment not influenced by anthropogenic resources, would not be considered to be peridomestic. Peridomestic mammals can be found nearly worldwide. 


\subsection{Spillover Hosts}

1.3 Bridge Hosts
For example, various mice and rat species, some of which have a nearly global distribution in terrestrial habitats, are often found in peridomestic settings. Bank voles (Myodes glareolus), which are commonly found in Asia and Europe, are often found in these settings as well [1]. Common raccoons (Procyon lotor), which are primarily found in North and Central America, but with introduced populations in Europe and Japan [2, 3], often exhibit peridomestic tendencies within urban and rural settings. The preceding examples represent a small subset of mammalian species that have peridomestic tendencies throughout the Old and New Worlds. While peridomestic mammals might play a broad role in general influenza A virus (IAV) ecology, current research has generally been focused on elucidating the role of these animals in avian IAV ecology and is the primary focus of this chapter.

Pathogen spillover processes relate to the dynamics that permit a pathogen to be transferred into an atypical host population from a reservoir (e.g., maintenance) host population [4]. A spillover event occurs when a pathogen of interest moves from one species into another [5]. Thus, for IAVs, a spillover event could be defined as occurring when an IAV moves from a reservoir or maintenance species into new, atypical, and/or non-maintenance host. If the process is successful and a productive infection ensues, the recipient species would be considered a spillover host.

Recent field and laboratory studies have indicated that some peridomestic mammal species are naturally exposed to or can replicate several IAVs, including those of avian origin in some instances. Examples can be found in raccoons [6], striped skunks (Mephitis mephitis) [7], cottontail rabbits (Sylvilagus sp.) [8], plateau pika (Ochotona curzoniae) [9], bank voles [1], and house mice (Mus musculus) [10]. Although some mammalian species, such as dogs, swine, and horses, are associated with endemic IAV strains (i.e., canine, swine, and equine influenza) that are readily transmitted among conspecifics, sustained transmission among the peridomestic mammals discussed in this chapter has not been documented. The potential maintenance of an IAV in raccoon populations was recently suggested in Japan [11], but additional work is needed to confirm this possibility which was hypothesized based on a small sample. Thus, some of the mammals listed above are potential spillover hosts that could acquire an IAV infection through interactions with waterfowl through predation, the use of common foraging sites, from potentially contaminated environments (e.g., by means of exposure to carcass compost areas of an infected poultry farm), or from virus-contaminated water sources.

The bridge host concept is a relatively recent addition to disease ecology. Bridge hosts are species that have the potential to interact with both maintenance hosts (hosts that maintain a pathogen) and target hosts (the population of concern) and meet several other 
criteria [12]. For IAVs, maintenance and target hosts are often thought of as waterfowl and/or their habitat and poultry or areas near the facilities (commercial or backyard) in which they are housed, respectively. In the case of IAVs and peridomestic mammals, the aforementioned criteria include species that can replicate and shed these viruses in moderate to large quantities or have the capacity to mechanically transmit these viruses [12]. For some mammalian species, the potential to interact (directly or indirectly) with both maintenance and target host populations is fairly obvious, as some species with a natural affinity for aquatic habitats are also attracted to various features of a poultry farm (e.g., compost, carcasses, spilled feed, etc.).

\subsection{Objective}

The potential role of peridomestic mammals in IAV ecology and epidemiology has received limited attention. Whether or not these species are relatively rare or relatively common bridge or spillover hosts has yet to be rigorously evaluated. If these host designations are common, some of these species could pose a potential biosecurity risk to poultry and livestock operations. Herein, we review challenges to working with these species, laboratory methods, and field sampling, with a focus on peridomestic mammalian species known to shed or be exposed to IAVs.

\section{Considerations/Challenges for Working with Influenza A Virus in Peridomestic Mammals}

\subsection{Infection Susceptibilities}

The general susceptibility of peridomestic mammals to IAVs is unknown for most species that fall into this category. Further, as is common with IAVs, susceptibility of a particular mammalian species to one strain or subtype of IAV does not necessarily translate to that of other subtypes so multiple studies need to be conducted before any generalizations can be made. Although it has been suggested that some avian IAVs may have limited capacity for replication in mammals, exceptions to this generality have been documented. For example, while some workers found that clade 2.3.4.4 goose/Guangdong/1996 lineage highly pathogenic (HP) avian IAVs have limited capacity for replication in mice and ferrets [13], moderate to moderately high levels of replication were found in peridomestic cottontail rabbits [14]. An additional consideration to understanding susceptibilities to IAVs for peridomestic mammals is infectious dose and route of infection. This topic has rarely been studied in peridomestic mammals, but is critical for assessing the role these species may play in IAV dynamics. For obvious reasons, the epidemiological implications of a replication competent host species are more impactful if the infectious dose is low and if that species is susceptible to infection from transmission routes associated with its normal behavior. In a dose response study assessing both the oral and nasal routes of infection in cottontail 


\subsection{Sampling Constraints}

\subsection{Zoonoses}

rabbits [15], the nasal route of infection was the most efficient, as a $100 \%$ infection rate was noted across all tested doses (as low as approximately $10^{2} \mathrm{EID}_{50}$ ). The oral route of infection was not productive at the low dose mentioned above, but still produced productive infections in some animals at relatively low doses (as low as approximately $10^{3} \mathrm{EID}_{50}$ ). The results of this study indicate that this peridomestic mammal is highly susceptible to an IAV with low infectious doses that are routinely observed in environments contaminated by maintenance hosts [15], as artificial ponds populated with experimentally infected mallards readily developed water titers of $>10^{3} \mathrm{PFU} / \mathrm{mL}$ [16]. Further, this study has implications for biosecurity at live bird markets, as rabbits are commonly found in live bird markets in many countries, including the USA.

Acquiring samples from wild terrestrial peridomestic mammals can be more costly in terms of time and money compared to birds. For example, obtaining biological samples from many mammalian species requires the use of anesthesia for the safety of both the animal and the handler. Obtaining and storing chemical immobilization agents generally requires a permit and using such agents often requires the user to be certified or to use the chemicals under the direction of a veterinarian. Additionally, chemically immobilizing animals can greatly increase the amount of time required to process a single individual since induction time can be lengthy and the individual must be monitored until it recovers, which can also take a considerable amount of time. Thus, sampling mammals is typically less efficient than sampling birds. In addition, field studies that target multiple peridomestic mammals require many sizes of live traps to capture the diversity of mammals that might occupy a particular site. These traps can be relatively expensive to obtain and can be bulky and are labor intensive to transport, set, maintain, and retrieve. Moreover, the wide range of mammalian activity patterns (i.e., diurnal, crepuscular, or nocturnal) may require that traps are monitored across all daylight hours.

The likely potential for zoonotic agents is an additional facet that must be taken into consideration when sampling mammals for IAVs. Although birds can also harbor zoonotic pathogens, including zoonotic IAVs, many more zoonotic agents are harbored by mammals as compared to birds. Some well-known examples include rabies virus, hantaviruses, and other pathogens that may be unique to mammals. Of significance, some of these pathogens have very high human morbidity and mortality rates. As such, various precautions such as vaccinations (when available) and personal protective equipment (e.g., leather gloves and possibly additional PPE dependent on the species sampled) and sample treatment (e.g., heat treatment of serum samples to neutralize adventitious pathogens that are potentially present) should be given strong consideration. 
2.4 Sample Types, Collection, and Handling
Samples typically collected from peridomestic mammals will vary due to study objectives, but in general IAV is a respiratory pathogen in mammals so samples targeting respiratory mucosa in live animals or respiratory tissue at necropsy are the most appropriate. In our experience sampling peridomestic mammals for IAVs, nasal wash or flush samples produce the highest titers of any antemortem sample that is practical for wildlife (i.e., compared to nasal swabs, oral/ tracheal swabs, and fecal swabs). Nasal washes can be accomplished by pipetting viral transport media in and out of the nares. Alternatively, it is often possible to elicit a sneeze during this process by prickling the nares while spritzing a viral transport media into the nasal cavity. In these instances, a petri dish is placed under/in front of the mammal's nose to collect expelled fluids. Nasal washes and/or nasal swabs are typically not practical to conduct on live small mammals such as mice. However, some workers have had success in IAV detection by harvesting and assaying respiratory tissues from house mice and Norway rats (Rattus norvegicus) $[10,17,23]$. In addition, respiratory tissues can be used to elucidate whether or not infections are primarily in the upper or lower respiratory tract. Oral swabs can also be a useful sample for some species (e.g., cottontail rabbits and striped skunks), but typically yields are decreased at least one order of magnitude as compared to nasal samples. Further, we have observed that detectable virus often wanes more rapidly in the oral cavity compared to the nasal cavity. Considering that the size of mammal species targeted for investigations can range from small rodents to mesocarnivores or even larger mammals in some instances, it is important to have multiple swab sizes available prior to sampling. In addition, the volume of viral transport media may need to be adjusted if multiple sizes of mammals are targeted. For example, if very limited sampling material is obtainable, a small media volume may be warranted as to not over dilute the material prior to conducting laboratory assays. Please refer to Chapters 9, 19, and 27 of this book for cold chain and transport guidelines, which can be adopted for sampling peridomestic mammals.

\section{Laboratory Methods}

3.1 Limited Species-Specific Methods
Unlike other animals (e.g., livestock and humans), species-specific laboratory methods for IAV detection and serology have not been developed, optimized, or validated for most wild peridomestic mammalian species. Thus, laboratory tests developed for other species are generally applied or adapted for testing peridomestic mammal samples for IAV or IAV-specific antibodies. Consequently, results from these tests must be interpreted with caution when used on sample types outside the range for which the tests were developed. An additional complication is that peridomestic mammals 


\subsection{Detection/ Culture Methods}

3.3 Serological Methods could be exposed to either avian or mammalian strains (e.g., swine, canine, equine) of IAVs so researchers need to carefully select appropriate versions of laboratory tests to address their specific objectives and to constrain inferences for any strain or subtypespecific tests.

RT-PCR targeting conserved regions of the IAV matrix gene have generally been shown to work across species. Matrix RT-PCR $[18,19]$ has been used with good success to detect viral RNA from nasal samples, oral samples, and/or tissues samples collected from several peridomestic mammals in experimental studies $[1,7$, $8,10,17,20]$, including bank voles, raccoons, striped skunks, cottontail rabbits, house mice, and Norway rats, and RT-PCR has been successfully used for molecular subtyping of plateau pika [21]. In addition, plaque assays have been used successfully to assess live virus from experimentally infected raccoons, striped skunks, and cottontail rabbits for nasal washes and oral swabs $[14,22]$, and virus isolation using standard methods in SPF eggs has been used on samples from raccoons, striped skunks, cottontail rabbits, bank voles, and Norway rats [1, 7, 8, 20, 23]. However, relatively low isolation rates were observed for some species [1]. Hemagglutination inhibition (HI) and neuraminidase inhibition (NI) assays for viral detection have been less commonly applied to samples from peridomestic mammals. However, they have been used to successfully detect IAVs in pika and raccoon dogs (Nyctereutes procyonoides) $[9,24]$.

Various serological tests have been used to assess antibodies to IAVs in peridomestic and other mammalian species (e.g., ELISA, AGID, HI, NI). For most of these tests, however, a criterion for a positive sample has not been well-established so careful consideration must be adopted before specifying positive and negative samples. Moreover, various constituents and inhibitors may be present in speciesspecific serum samples that invalidate the use of a particular test on a given species. In these cases, samples from experimentally infected animals and known positive animals are critical for rigorous evaluation of serological tests.

A variety of ELISA assays have been used on peridomestic mammal serum samples. The IDEXX MultiS-Screen AB test (an ELISA), as one example, has been validated by the manufacturer for various poultry species. The manufacturer suggests a cutoff value of a sample-to-negative $(\mathrm{S} / \mathrm{N})$ value of 0.5 for the evaluated avian species. Others have evaluated this test with swine serum and found the optimized $\mathrm{S} / \mathrm{N}$ value to be 0.673 for this species, which they were able to establish due to access to a large number of archived serum samples from experimentally infected and vaccinated swine [25]. It is unlikely that there will be a sufficient number of serum samples collected from experimentally 
infected peridomestic mammals for most species to conduct this type of an analysis to identify an appropriate cutoff threshold for individual species. Thus, some researchers have used the manufacturers' (avian) cutoff value to assess IAV exposures in field samples collected from various mammalian species [26]. In experimental settings, others have used the change in $\mathrm{S} / \mathrm{N}$ values in pre- versus post-experimental infection serum samples to infer seroconversion using this ELISA [7].

Although the IDEXX ELISA performed well with raccoon and striped skunk sera from experimentally infected animals $[7,20]$, this assay did not work on cottontail rabbit sera following experimental infection studies [8]. The reason for this is unclear, but this information would not have been known without the use of known samples collected from experimental infection studies. Other ELISAs have also been used for testing of peridomestic mammal serum samples with various tests used for bank voles and rats in addition to an in-house indirect ELISA that worked on house mouse samples $[1,10,23,27]$.

The agar immunodiffusion (AGID) test is another serological assay that has been commonly used on peridomestic mammal samples. While the IDEXX ELISA did not perform appropriately for cottontail rabbits, AGID tests showed good concordance with infection status and seroconversion in this species [8, 15]. AGID tests have also been used on serum samples collected from raccoons and skunks $[6,7,28]$.

HI tests have been commonly used serological tests and have been used to test bank voles, raccoons, skunks, cottontail rabbits, and plateau pika, with NI tests used on raccoons and plateau pika $[1,2,9,22,28]$. These tests have the added advantage that they can identify specific subtypes, but they require reference viruses which is not problematic for experimental studies in which the test virus can be used, but for field studies reference viruses must be carefully selected to match study objectives or some exposures may be missed. Additionally, serum inhibitors can be a problem for $\mathrm{HI}$ testing of mammalian samples so samples must be treated (e.g., with receptor-destroying enzyme) to inactivate inhibitors [29]. A final consideration is erythrocyte source for these tests. Chicken red blood cells are commonly used, but in some cases turkey or equine erythrocytes may be more appropriate.

Horimoto et al. [2] and Yamaguchi et al. [30] used virus neutralization tests with raccoon specimens for subtype-specific antibody detection and Yu et al. [31] used a similar assay in plateau pika. Microneutralization assays have been recently shown to be more readily able to detect antibodies to IAVs in avian serum when serum samples were collected 2 weeks postinfection as compared to those sampled 4 weeks postinfection [32]. Perhaps this or similar techniques could be useful when peridomestic mammal exposure to IAVs is presumed to have been recent, such as at an outbreak. 
3.4 Additional Laboratory Methods
Other standard IAV laboratory tools that have been used on peridomestic mammals include immunohistochemistry for bank voles and plateau pika and basic histology in bank voles, raccoons, striped skunks, and cottontail rabbits [1, 22, 33].

\section{Field Methods}

\subsection{Field Studies}

Understanding the role peridomestic mammals may play in either the natural ecology of IAVs or at the livestock or human interfaces requires systematic field studies. To date, field studies of IAV infections in mammals have been fairly rare and documentation of shedding or exposure has yet to be rigorously assessed for most peridomestic mammalian species. The plateau pika in China and raccoons in the USA and Japan are two species that have received at least some attention on this topic.

Antibodies to IAVs have been reported in raccoons from multiple states in the U.S $[6,28]$. This initial finding was followed by work assessing IAV exposure in invasive raccoon populations in Japan. Exposures to multiple IAV subtypes have been reported in both countries [2, 6, 28, 30]. More recently, IAV genes were detected in samples collected from raccoons in Japan [11].

The plateau pika represents one of the most intensively studied peridomestic mammals in terms of IAV associations. Multiple studies have been conducted in the Qinghai region of China, which is an area where IAV has received significant attention due to its status as a congregation site for migrating waterfowl, especially bar-headed geese (Anser indicus) which were associated with the initial emergence of Asian HP H5Nl IAV. Evidence of exposure to multiple virus subtypes, such as $\mathrm{H} 5 \mathrm{~N} 1, \mathrm{H} 9 \mathrm{~N} 2$, and $\mathrm{H} 7 \mathrm{~N} 2$, have been reported for wild plateau pika that regularly share habitat with waterfowl $[9,21,31,34]$. Researchers have suggested the transmission of IAV from waterfowl to pika likely occurred through shared virus-contaminated food resources [9]. Experimental studies have demonstrated that this species is susceptible to infection with multiple IAV subtypes, such as $\mathrm{H} 1 \mathrm{~N} 1, \mathrm{H} 3 \mathrm{~N} 2$, and $\mathrm{H} 5 \mathrm{Nl}$ [33]. These studies have motivated several experimental studies of IAVs in North American lagomorphs (i.e., rabbits, hares, and pika) to assess susceptibility and shedding $[8,14,15,22]$.

In addition to studying peridomestic mammals in wild habitats, assessing their importance for farm biosecurity also requires IAV surveillance at agricultural facilities such as poultry farms. Of key importance is sampling these operations during active outbreaks of agriculturally relevant IAV strains such as $\mathrm{H} 5$ and $\mathrm{H} 7$ viruses.

Several considerations must be taken into account when conducting surveillance on peridomestic mammals at outbreak sites, one of which is seasonal differences in behavior. For example, 
periods of dispersal of young animals could be periods of increased capture success. Furthermore, these periods of dispersal could increase the risk of long-distance IAV trafficking by some species. An additional issue associated with seasonality is that capture and handling approaches may differ by time of year because behavior changes across seasons.

The size of target mammals is an additional consideration of importance. Generically speaking, there will generally be three types of mammals that utilize poultry farms in one fashion or another. These include small mammals such a rodents, which in many instances can readily enter a poultry barn and may or may not move from barn to barn. The risk posed by this order of mammals can be from replication of the virus in question or as a potential mechanical vector [35]. Slightly larger mammals, such as cottontail rabbits, could readily move from barn to barn, forage in the same areas as select wild waterfowl such as geese (e.g., Branta spp. and Chen spp.), but are unlikely to have the capacity to enter a barn under normal conditions. The third mammal type likely to utilize poultry farms can be found in mesocarnivores. These species, which will often be represented by raccoons, skunks, and other small carnivores, have the capacity to move from farm to farm but incursions into barns are unlikely for these larger species under normal circumstances. Instances when these larger mammals could breach building interiors of a poultry farm would most likely be associated with a door inadvertently left open or damage to the facility of sufficient size for the species in question to negotiate the obstacle. Unfortunately, it is generally difficult to obtain optimal sample sizes of mesocarnivores at outbreak sites unless a large number of farms are sampled.

To date, only limited work has been conducted on peridomestic mammals associated with IAV infections at poultry facilities. Some recently published examples were linked with the 2015 outbreak of clade 2.3.4.4 highly pathogenic IAVs in poultry facilities in the USA $[36,37]$. While these studies did not identify exposure or infection in any mammal species tested, both studies were conducted weeks to months after outbreaks. Real-time surveillance of peridomestic mammals during outbreaks will provide the best information for assessing potential risks associated with these species and should be a high priority for future studies.

\subsection{Alternative Surveillance Methods}

Aside from taking biological samples from peridomestic mammals near poultry farms, some additional techniques common in the field of wildlife biology are useful for characterizing behavioral and space use patterns of mammalian species. Mark-recapture studies, as one example, could be used to assess if small rodents move from the inside to the outside of a poultry barn or between barns. This same technique could be used to assess whether mesocarnivores utilize elements of poultry facilities but also travel to areas 


\subsection{Ecological "Hotspots"}

where waterfowl might be present, such as ponds, lakes, wetlands, and crop fields or whether these species move between farms.

Camera traps represent an additional and more passive type of surveillance that could be used to provide an inventory of the peridomestic mammals that occur on and near poultry production facilities. For example, a recent study identified over 40 mammalian and avian species that visited mortality pits (e.g., burial pits for animal carcasses) in Colorado [38]. Similar techniques could be used at key areas of poultry farms (e.g., compost bins, feed storage areas, etc.) to assess mammalian species that commonly visit poultry farms. Notably, work of this type identified four medium-sized mammals (raccoon, Virginia opossum [Didelphis virginiana], striped skunk, and domestic cat [Felis catus]) that visited composting sites on poultry facilities [36].

The identification of and sampling at key sites (e.g., ecological "hotspots") that contain large numbers of avian IAV hosts and habitat suitable for select mammal species, especially those that spatially overlap poultry facilities or other livestock facilities, is an important area of future research for mammalian associations with IAVs. For example, the small number of IAV isolates detected from peridomestic mammals is likely, in part, due to the fact that sampling for peridomestic mammals outside of active poultry outbreaks is relatively rare. Qinghai Lake is somewhat unique in that it is a consistent "hotspot" for IAV activity in wild birds, thereby giving the local mammalian fauna ample opportunities to directly and indirectly interact with avian hosts that are shedding virus and contaminating shared environments. Perhaps avian IAV hotspots elsewhere, such as the Delaware bay in the USA [39], might yield similar results if active IAV surveillance is undertaken in key mammalian species at this and similar locations.

\section{Approaches to Designing Studies to Investigate Influenza A Virus in Peridomestic Mammals}

\subsection{Target Species}

Approaches to studying IAV in peridomestic mammals will be largely dependent on the study objectives. However, a question that should be addressed initially is how are the species that are being targeted relevant to IAV epidemiology? In an outbreak situation, relevant species would include those that have the potential to visit areas with both aquatic habitats and poultry farms (e.g., select mesocarnivores), those commonly associated with farms (e.g., rabbits), and those that are small enough in size to regularly infiltrate poultry barns (e.g., small rodents). In regard to surveillance of IAVs in peridomestic mammals during non-outbreak periods, the most relevant species are those that directly or indirectly interact with waterfowl. The common thread in each of these scenarios is 
likely areas where mammals and waterfowl might interact, such as water bodies or common feeding sites. For example, a recent publication indicated that IAV outbreaks at poultry farms with nearby water bodies exhibited higher incidence [40]. However, surveillance of this type has been rarely undertaken with mammals.

An additional consideration (when data are available) is that a species, especially one that visits aquatic habitats, could show regular evidence of exposure, but shed no or only limited quantities of virus. A good example of this scenario can be found in the raccoon. Raccoons have been shown to commonly have IAV antibodies in some parts of the USA as well as in introduced populations in Japan $[2,6,30]$. However, experimental studies have indicated that they typically shed low to moderate quantities of virus $[6,20]$, although moderately high shedding was detected in some individuals for one of the tested strains [22]. As a comparison, striped skunks have been shown to shed higher quantities of virus, occasionally for extended periods $[7,22]$, and can likely replicate multiple strains from multiple origins $[7,41]$, but aside from some field studies associated with pandemic $\mathrm{HINl}$ [41], no thorough evaluations of IAV associations in striped skunks in non-laboratory settings have been published. Alternatively, an animal that exhibits a productive infection in the laboratory may only rarely or never be exposed to IAVs in natural settings. Thus, in many instances, the most useful information will come from a combination of both field and laboratory studies that examine natural exposure rates and the replication/shedding potential of these types of mammals.

5.2 Limitations to Sampling Small Mammalian Species
Rodents are likely the mammals that will be captured in the largest numbers during most investigations, especially those involving poultry facilities. Nonetheless, their typical small size will often limit the samples that can be acquired from them. For example, conducting a nasal wash or nasal swab on a small rodent is generally not feasible due their small size, but is typically the best sample that can be collected from a live mammal. Notably, titers from postmortem nasal washes of experimentally infected house mice were typically orders of magnitude lower than select tissues harvested from this species [10]. Further, oral swab samples were only rarely positive in this species and, when positive, were so at very low levels. With these constraints in mind, select tissues (e.g., lungs, nasal turbinates, and trachea) may represent the best target sample when addressing small rodents. An additional consideration when sampling small rodents is that rapid population turnover can occur frequently, which reduces the probability that sampled animals had direct exposure during an outbreak. 


\section{Summary}

While a number of wild mammals have been shown to be naturally exposed to IAVs, the role of most of these species in the epidemiology of these viruses remains to be determined. Additional field work coupled with experimental infection and experimental transmission studies are needed to assess which species represent a true risk to agriculture and/or zoonotic infections versus those that may be exposed to these IAVs but contribute little to their transmission.

\section{References}

1. Romero Tejeda A, Aiello R, Salomoni A, Berton V, Vascellari M, Cattoli G (2015) Susceptibility to and transmission of $\mathrm{H} 5 \mathrm{Nl}$ and $\mathrm{H} 7 \mathrm{Nl}$ highly pathogenic avian influenza viruses in bank voles (Myodes glareolus). Vet Res 46:51

2. Horimoto T, Maeda K, Murakami S, Kiso M, Iwatsuki-Horimoto $\mathrm{K}$, Sashika M, Ito $\mathrm{T}$, Suzuki K, Yokoyama M, Kawaoka Y (2011) Highly pathogenic avian influenza virus infection in feral raccoons, Japan. Emerg Infect Dis 17:714-717

3. Mori E, Mazza G, Menchetti M, Panzeri M, Gager Y, Bertolino S, Di Febbraro M (2015) The masked invader strikes again: The conquest of Italy by the Northern raccoon. Hystrix 26:1-5

4. Hassell JM, Begon M, Ward MJ, Fèvre EM (2017) Urbanization and Disease Emergence: Dynamics at the Wildlife-Livestock-Human Interface. Trends Ecol Evol 32:55-67

5. Brown C (2013) Spillover: Animal infection and the next human pandemic. Emerg Infect Dis 19:349

6. Hall JS, Bentler KT, Landolt G, Elmore SA, Minnis RB, Campbell TA, Barras SC, Root JJ, Pilon J, Pabilonia K, Driscoll C, Slate D, Sullivan H, McLean RG (2008) Influenza infection in wild raccoons. Emerg Infect Dis 14:1842-1848

7. Root JJ, Shriner SA, Bentler KT, Gidlewski T, Mooers NL, Ellis JW, Spraker TR, VanDalen KK, Sullivan HJ, Franklin AB (2014) Extended viral shedding of a low pathogenic avian influenza virus by striped skunks (Mephitis mephitis). PLoS One 9:e70639

8. Root JJ, Shriner SA, Bentler KT, Gidlewski T, Mooers NL, Spraker TR, VanDalen KK, Sullivan $H J$, Franklin AB (2014) Shedding of a low pathogenic avian influenza virus in a common synanthropic mammal - the cottontail rabbit. PLoS One 9:e103513
9. Zhou J, Sun W, Wang J, Guo J, Yin W, Wu N, Li L, Yan Y, Liao M, Huang Y, Luo K, Jiang X, Chen $\mathrm{H}(2009)$ Characterization of the $\mathrm{H} 5 \mathrm{Nl}$ highly pathogenic avian influenza virus derived from wild pikas in China. J Virol 83:8957-8964

10. Shriner SA, VanDalen KK, Mooers NL, Ellis JW, Sullivan HJ, Root JJ, Franklin AB (2012) Low-pathogenic avian influenza viruses in wild house mice. PLoS One 7:e39206

11. Yamaguchi E, Fujii K, Ogawa $\mathrm{H}$, Imai $\mathrm{K}$ (2018) First detection of influenza A virus genes from wild raccoons in Japan. Virus Genes 54:591-595

12. Caron A, Cappelle J, Cumming GS, de GarineWichatitsky M, Gaidet N (2015) Bridge hosts, a missing link for disease ecology in multi-host systems. Vet Res 46:83

13. Kaplan BS, Russier M, Jeevan T, Marathe B, Govorkova EA, Russell CJ, Kim-Torchetti M, Choi YK, Brown I, Saito T, Stallknecht DE, Krauss S, Webby RJ (2016) Novel highly pathogenic avian $\mathrm{A}(\mathrm{H} 5 \mathrm{~N} 2)$ and $\mathrm{A}(\mathrm{H} 5 \mathrm{~N} 8)$ influenza viruses of clade 2.3.4.4 from North America have limited capacity for replication and transmission in mammals. mSphere 1 : e00003-e00016

14. Root JJ, Bosco AM, Marlenee NL, Bowen RA (2018) Cottontail rabbits shed clade 2.3.4.4 H5 highly pathogenic avian influenza A viruses. Arch Virol 163:2823-2827

15. Root JJ, Shriner SA, Ellis JW, VanDalen KK, Sullivan HJ (2017) Low viral doses are sufficient to infect cottontail rabbits with avian influenza A virus. Arch Virol 162:3381-3388

16. Achenbach JE, Bowen RA (2011) Transmission of avian influenza A viruses among species in an artificial barnyard. PLoS One 6:e17643

17. Cummings CO, Hill NJ, Puryear WB, Rogers B, Mukherjee J, Rosenbaum $\mathrm{MH}$, Rustlander JA (2019) Evidence of influenza A 
in wild Norway rats (Rattus norvegicus) in Boston, Massachusetts. Front Ecol Evol 7:36

18. Runstadler JA, Happ GM, Slemons RD, Sheng ZM, Gundlach N, Petrula M, Senne D, Nolting J, Evers DL, Modrell A, Huson H, Hills S, Rothe T, Marr T, Taubenberger JK (2007) Using RRT-PCR analysis and virus isolation to determine the prevalence of avian influenza virus infections in ducks at Minto Flats State Game Refuge, Alaska, during August 2005. Arch Virol 152:1901-1910

19. Spackman E, Senne DA, Bulaga LL, Myers TJ, Perdue ML, Garber LP, Lohman K, Daum LT, Suarez DL (2003) Development of real-time RT-PCR for the detection of avian influenza virus. Avian Dis 47:1079-1082

20. Root JJ, Bentler KT, Shriner SA, Mooers NL, VanDalen KK, Sullivan HJ, Franklin AB (2014) Ecological routes of avian influenza virus transmission to a common mesopredator: an experimental evaluation of alternatives. PLoS One 9: e102964

21. Su S, Xing G, Wang J, Li Z, Gu J, Yan L, Lei J, Ji S, Hu B, Gray GC, Yan Y, Zhou J (2016) Characterization of $\mathrm{H} 7 \mathrm{~N} 2$ avian influenza virus in wild birds and pikas in Qinghai-Tibet Plateau area. Sci Rep 6:30974

22. Root JJ, Bosco-Lauth AM, BielefeldtOhmann H, Bowen RA (2016) Experimental infection of peridomestic mammals with emergent H7N9 (A/Anhui/1/2013) influenza A virus: implications for biosecurity and wet markets. Virology 487:242-248

23. VanDalen KK, Nemeth NM, Thomas NO, Barrett NL, Ellis JW, Franklin AB, Shriner SA (2019) Experimental infections of Norway rats with avian-derived low pathogenic influenza A viruses. Arch Virol 164:1831-1836

24. Qi X, Li X, Rider P, Fan W, Gu H, Xu L, Yang Y, Lu S, Wang H, Liu F (2009) Molecular characterization of highly pathogenic $\mathrm{H} 5 \mathrm{Nl}$ avian influenza A viruses isolated from raccoon dogs in China. PLoS One 4:e4682

25. Ciacci-Zanella JR, Vincent AL, Prickett JR, Zimmerman SM, Zimmerman JJ (2010) Detection of anti-influenza A nucleoprotein antibodies in pigs using a commercial influenza epitope-blocking enzyme-linked immunosorbent assay developed for avian species. J Vet Diagn Invest 22:3-9

26. Van Hemert C, Spivey TJ, Uher-Koch BD, Atwood TC, Sinnett DR, Meixell BW, Hupp JW, Jiang K, Adamas LG, Gustine DD, Ramey AM, Wan XF (2019) Survey of arctic Alaskan wildlife for influenza A antibodies: limited evidence for exposure of mammals. J Wildl Dis 55:387-398
27. Sullivan HJ, Blitvich BJ, VanDalen K, Bentler KT, Franklin AB, Root JJ (2009) Evaluation of an epitope-blocking enzyme-linked immunosorbent assay for the detection of antibodies to influenza A virus in domestic and wild avian and mammalian species. J Virol Methods 161:141-146

28. Roberts NM, Henzler DJ, Clark L (2009) Serologic evidence of avian influenza (H4N6) exposure in a wild-caught raccoon. Avian Dis 53:455-457

29. Cwach KT, Sandbulte HR, Klonoski JM, Huber VC (2012) Contribution of murine innate serum inhibitors toward interference within influenza virus immune assays. Influenza Other Respir Viruses 6:127-135

30. Yamaguchi E, Sashika M, Fujii K, Kobayashi K, Bui VN, Ogawa H, Imai K (2014) Prevalence of multiple subtypes of influenza A virus in Japanese wild raccoons. Virus Res 189:8-13

31. Yu Z, Cheng K, Sun W, Xin Y, Cai J, Ma R, Zhao Q, Li L, Huang J, Sang X, Li X, Zhang K, Wang T, Qin C, Qian J, Gao Y, Xia X (2014) Lowly pathogenic avian influenza (H9N2) infection in plateau pika (Ochotona curzoniae), Qinghai Lake, China. Vet Microbiol 173:132-135

32. Segovia KM, França MS, Bahnson CS, LatorreMargalef N, Stallknecht DE (2018) Are microneutralization and hemagglutination inhibition assays comparable? Serological results from influenza experimentally infected mallard ducks. Avian Dis 63:138-144

33. Li Y, Xiao H, Huang C, Sun H, Li L, Su J, Ma J, Liu D, Wang H, Liu W, Gao GF, Li X, Yan J (2015) Distribution of sialic acid receptors and experimental infections with different subtypes of influenza A viruses in QinghaiTibet plateau wild pika. Virol J 12:63

34. Yan Y, Gu JY, Yuan ZC, Chen XY, Li ZK, Lei J, Hu BL, Yan LP, Xing G, Liao M, Zhou JY (2017) Genetic characterization of H9N2 avian influenza virus in plateau pikas in the Qinghai Lake region of China. Arch Virol 162:1025-1029

35. Velkers FC, Blokhuis SJ, Veldhuis Kroeze EJB, Burt SA (2017) The role of rodents in avian influenza outbreaks in poultry farms: A review. Vet Q 37:182-194

36. Grear DA, Dusek RJ, Walsh DP, Hall JS (2017) No evidence of infection or exposure to highly pathogenic avian influenzas in peridomestic wildlife on an affected poultry facility. J Wildl Dis 53:37-45

37. Shriner SA, Root JJ, Lutman MW, Kloft JM, VanDalen KK, Sullivan HJ, White TS, Milleson MP, Chandler SC, Wolf PC, Turnage CT, 
McCluskey BJ, Vincent AL, Torchetti MK, Gidlewski T, Deliberto TJ (2016) Surveillance for highly pathogenic $\mathrm{H} 5$ avian influenza A virus in synanthropic wildlife associated with poultry facilities during an acute outbreak. Sci Rep 6:36237

38. Ellis JW, Shriner SA, McLean HE, Petersen L, Root JJ (2017) Inventory of wildlife use of mortality pits as feeding sites: Implications of pathogen exposure. Hum Wildl Interact 11:8-18

39. Brown VL, Drake JM, Stallknecht DE, Brown JD, Pedersen K, Rohani P (2013) Dissecting a wildlife disease hotspot: the impact of multiple host species, environmental transmission and seasonality in migration, breeding and mortality. J R Soc Interface 10:20120804

40. Shimizu Y, Hayama Y, Yamamoto T, Murai K, Tsutsui T (2018) Matched case-control study of the influence of inland waters surrounding poultry farms on avian influenza outbreaks in Japan. Sci Rep 8:3306

41. Britton AP, Sojonky KR, Scouras AP, Bidulka JJ (2010) Pandemic (HINl) 2009 in skunks, Canada. Emerg Infect Dis 16:1043-1045 